\title{
The differential cell signaling effects of two positional isomers of the anticancer NO-donating aspirin
}

\author{
AMY HUA* , GERARDO G. MACKENZIE* and BASIL RIGAS \\ Division of Cancer Prevention, Department of Medicine, Stony Brook University, \\ Stony Brook, New York 11794-5200, USA
}

Received May 19, 2009; Accepted June 22, 2009

DOI: 10.3892/ijo_00000397

\begin{abstract}
We studied the mechanism by which the para and meta positional isomers of nitric oxide-donating aspirin (NO-ASA) inhibit human colon cancer cell growth. These compounds are promising chemopreventive agents and represent a broader class of novel drugs. The two isomers differ drastically in their $24-\mathrm{h} \mathrm{IC}_{50} \mathrm{~s}$ for cell growth, which are $12 \mu \mathrm{M}$ for $p$-NO-ASA and $230 \mu \mathrm{M}$ for $m$-NO-ASA. We examined their effects on cell signaling cascades, including predominantly the mitogen activated protein kinases (MAPKs). The principal differences between the two isomers were: a) $p$-NO-ASA exerts its effect earlier than $m$-NO-ASA; b) the predominant effect of $m$-NO-ASA is on ERK1/2 and Akt; whereas that of $p$-NO-ASA is on JNK1/2, while both activate p38, with $p$-NO-ASA showing a stronger and earlier effect; c) ATF-2 is more responsive to $m$-NO-ASA and c-Jun to $p$ NO-ASA; d) both isomers seem to have similar effects on AP-1 binding, the main difference between them being the timing of the effect; $p$-NO-ASA's effect is early and $m$-NOASA's is late; e) $p$-NO-ASA has an earlier and stronger effect on p21, while $m$-NO-ASA's effect occurs later and is weaker; and f) cell cycle changes follow the effect on p21 expression. Our findings underscore the role of positional isomerism in modulating the pharmacological effects of drugs and have potentially important implications for the further development of these chemoprevention agents.
\end{abstract}

Correspondence to: Professor Basil Rigas, Division of Cancer Prevention, Life Sciences Building, Room 06, Stony Brook University, Stony Brook, NY 11794-5200, USA

E-mail: basil.rigas@stonybrook.edu

*Contributed equally

Abbreviations: EMSA, electrophoretic mobility shift assay; MAPKs, mitogen activated protein kinases; NO-ASA, nitric oxidedonating aspirin

Key words: nitric oxide-donating aspirin, positional isomerism, p21, mitogen activated protein kinases, cancer prevention

\section{Introduction}

Colon cancer has the third highest cancer incidence in the United States, with over 50,000 individuals dying each year of causes related to colorectal cancer (1). These statistics reflect the limitations of currently available treatments. The development of new preventive protocols is essential to reduce the incidence of colon cancer. In the search of such approaches, aspirin and other non-steroidal antiinflammatory agents have shown promising results (2-4). The prolonged use of aspirin for cancer prevention in doses other than those used for cardiac prophylaxis, however, is not practical because of its toxicity $(5,6)$. Thus, new anti-inflammatory derivatives of aspirin, such as nitric oxide-donating aspirin (NO-ASA), are being explored. NO-ASA shows much promise because of its significantly lower gastrointestinal toxicity compared to aspirin (7) and its antineoplastic effects in preclinical models of colon cancer (8-10); for example, NO-ASA inhibits the growth of cultured pancreatic and colon cancer cells $(8,11)$, as well as intestinal carcinogenesis in Min mice (9) and pancreatic cancer in hamsters (12).

Isomerism is of particular interest to pharmacology as it can influence drug activity, at times profoundly (13-15). There are four main types of drug isomers, positional isomers, geometric isomers, enantiomers and diastereoisomers. Positional isomers are compounds of the same molecular formula but differ in the position of a functional group in the drug molecule. The ability of positional isomerism to modulate drug effect has been classically exemplified by the isomeric amobarbital and pentobarbital, in which the position of a methyl group markedly affects their pharmacological activity. NO-ASA has three positional isomers para, meta and ortho, based on the position of the $-\mathrm{ONO}_{2}$ group with respect to its ester bond (Fig. 1). We have reported that these positional isomers vary in their potency to inhibit the growth of colon cancer cells; ortho and para are nearly equipotent, whereas the meta is far less potent than the other two (16). We have explored several intracellular signaling pathways to determine how $p$-NO-ASA mediates its unique growth inhibitory effects $(10,17-21)$. Previously, we identified mitogen activated protein kinase (MAPK) pathways as mediators of $p$-NO-ASA's growth inhibitory effect (19). Employing two colon cancer and one pancreatic cell line we demonstrated that $p$-NO-ASA activated several MAPKs, and that JNK and p38 were required for its growth inhibitory effect (19). 
The present study examined whether the significant difference in the growth inhibitory potency of $m$ - and $p$-NOASA could be accounted for by differences in their intracellular signaling. Thus, we compared the signaling cascades activated by the isomers of NO-ASA, focusing on the MAPK signaling cascade and its dependent pathway that leads via p21 to cyclin D1 expression and cell cycle regulation. We demonstrated significant differences between the two isomers of NO-ASA in MAPK signaling, which led to differential induction of $\mathrm{p} 21$, which in turn affected cyclin D1 expression and cell cycle phase transitions.

\section{Materials and methods}

Materials. NO-ASA, both para [2-(acetyloxy) benzoic acid 4-(nitrooxy methl) phenyl ester] and meta [2-(acetyloxy) benzoic acid 3-(nitrooxy methl) phenyl ester], was synthesized as previously described (22). Antibodies against the phosphorylated forms of cJun, ATF-2, JNK, p38, ERK1/2, Akt and total p21 were purchased from Cell Signaling Technology, Beverly, MA. Horseradish peroxidase conjugated antibodies against $\alpha$-tubulin, mouse $\operatorname{IgG}$, or rabbit $\operatorname{IgG}$ were purchased from Oncogene Research Products, San Diego, CA. All siRNAs were obtained form Upstate Cell Signaling Solutions, Lake Placid, NY. Cell lines were obtained from American Type Culture Collection (ATCC), Manassas, VA and cell culture reagents from Mediatech, Herndon, VA.

Cell culture and treatments. Cells were grown to $80 \%$ confluence in the media recommended by ATCC supplemented with $10 \%$ fetal calf serum, 1,000 U/ml penicillin and $1,000 \mu \mathrm{g} / \mathrm{ml}$ streptomycin. For treatment with various reagents, $1 \times 10^{6}$ cells/plate were plated in 6 -well plates (Corning, Corning, NY). NO-ASA and other reagents were incubated with cell monolayers in the presence of $1 \%$ DMSO in culture medium. After incubation, cultures were placed on ice, the medium was collected and cells were washed twice with phosphate-buffered saline (PBS). Lysis buffer [20 mM HEPES, $50 \mathrm{mM} \mathrm{NaF}, 1 \mathrm{mM} \mathrm{Na}_{3} \mathrm{VO}_{4}, \mathrm{pH} 7.3$, with $10 \%$ (v/v) glycerol, $1 \%$ (v/v) Triton X-100, $20 \mu \mathrm{g} / \mathrm{ml}$ leupeptin, $20 \mu \mathrm{g} / \mathrm{ml}$ aprotinin, $1 \mathrm{mM}$ PMSF, and $2.5 \mathrm{mM}$ 4-nitrophenylphosphate] was added to cultures in wells; floating cells from the cultures were pooled with the adherent cells Protein concentration was determined using the BCA protein assay kit (Pierce, Rockford, IL).

Cell proliferation assay. Cell proliferation was assayed using the MTT colorimetric assay kit (Roche, Germany), following the manufacturer's instructions.

Western blots. Electrophoresis of cell lysates were performed on $10 \%$ SDS-PAGE gels as described (23); protein were electrophoretically transferred onto nitrocellulose membranes, which were reprobed as needed.

Isolation of nuclear extracts and electrophoretic mobility shift assays (EMSA). Nuclear extracts from cells cultured as above, were obtained as described (24), with the modifications listed below. Cells (approximately $1 \times 10^{7} / 100 \mathrm{~mm}$ dishe) were incubated on ice with whole cell fractionation buffer [10 $\mathrm{mM}$ HEPES, pH 7.9, $50 \mathrm{mM} \mathrm{NaF}, 1 \mathrm{mM} \mathrm{Na}_{3} \mathrm{VO}_{4}, 0.1 \mathrm{mM}$ EDTA,
$0.5 \mathrm{mM}$ DTT, $20 \mu \mathrm{g} / \mathrm{ml}$ leupeptin, $20 \mu \mathrm{g} / \mathrm{ml}$ aprotinin, $250 \mathrm{mM}$ 4-nitrophenylphosphate and $1 \mathrm{mM}$ PMSF] for $15 \mathrm{~min}$, then scraped and placed in chilled $1.5 \mathrm{ml}$ microfuge tubes on ice. To each $1.5 \mathrm{ml}$ suspension $0.15 \mathrm{ml}$ of $10 \%(\mathrm{v} / \mathrm{v}) \mathrm{NP}-40$ was added and the suspension was gently mixed. Tubes were centrifuged for $1 \mathrm{~min}$ at $4^{\circ} \mathrm{C}$, the supernatant was aspirated, $0.05 \mathrm{ml}$ of nuclear extraction buffer [20.0 mM HEPES, $\mathrm{pH} 7.9$, $25 \mathrm{mM} \mathrm{NaF}, 10 \mathrm{mM} \mathrm{Na}_{3} \mathrm{VO}_{4}, 0.1 \mathrm{mM}$ EDTA, $1.5 \mathrm{mM}$ $\mathrm{MgCl}_{2}, 420 \mathrm{mM} \mathrm{NaCl}, 20 \mu \mathrm{g} / \mathrm{ml}$ leupeptin, $20 \mu \mathrm{g} / \mathrm{ml}$ aprotinin, $250 \mathrm{mM}$ 4-nitrophenylphosphate, $1 \mathrm{mM}$ PMSF and 25\% (v/v) glycerol] was added, incubated for $30 \mathrm{~min}$ on a rocker with sporadic vortexing and centrifuged at $16,168 \mathrm{x}$ g for $10 \mathrm{~min}$ at $4^{\circ} \mathrm{C}$. The supernatants were collected as nuclear extracts, aliquoted and stored at $-80^{\circ} \mathrm{C}$ until analyzed.

EMSA. EMSA for transcription factor binding to the AP-1 site was performed using a non-radioactive EMSA kit from Panomics, Redwood, CA. Nuclear extracts $(10 \mu \mathrm{g})$ were incubated with an AP-1 botinylated probe for $30 \mathrm{~min}$ at room temperature. Excess cold AP-1 probe was added as a control. Separation of bound from unbound oligonucleotide probe was performed by electrophoresis in non-denaturing polyacrylamide gels and complexes were transferred to a nylon membrane. Biotin-labeled probe was visualized by enhanced chemiluminescence (ECL). The identification of transcription factors bound to the AP-1 probe was achieved by reprobing the nylon membranes with antibodies directed against cJun, ATF-2 and JunB to determine which transcription factors were bound to the AP-1 probe. This method was verified by incubating the nuclear extracts with a nonbiotinylated AP-1 probe and electrophoretic separation of complexes. The separated complexes were transferred to nylon membranes and the membranes were probed with antibodies to various transcription factors.

Cell transfections with siRNA. BxPC-3 cells in 6-well plates at $80 \%$ confluence were transfected with $200 \mathrm{ng}$ of a given siRNA suspended in $100 \mu 1$ Lipofectamine 2000 (Invitrogen, Carlsbad, CA). At $48 \mathrm{~h}$ post transfection, transfected cells were replated and treated with NO-ASA.

Evaluation of cell cycle progression. Cells $\left(2.0 \times 10^{6}\right.$ cells) were incubated in the presence of $m$ - or $p$-NO-ASA or equivalent volumes of DMSO for 4 and/or $24 \mathrm{~h}$. After each treatment, cells were trypsinized and fixed in 70\% (v/v) ethanol for $1 \mathrm{~h}$ on ice, stained with propidium iodide (PI) $(50 \mu \mathrm{g} / \mathrm{ml})$ and RNase A (4 U/ml) for 30 min and subjected to flow cytometry analysis.

Statistical analysis. Statistical evaluation of the data was performed by one-factor analysis of variance followed by Tukey test for multiple comparisons. $\mathrm{P}<0.05$ were regarded as statistically significant. Data presented were obtained from at least 3 independent experiments $(n=3)$ and are expressed as the mean \pm SEM.

\section{Results}

Both isomers of NO-ASA inhibit the growth of colon cancer cells. HT-29 cells were incubated with para or meta NO-ASA 
A

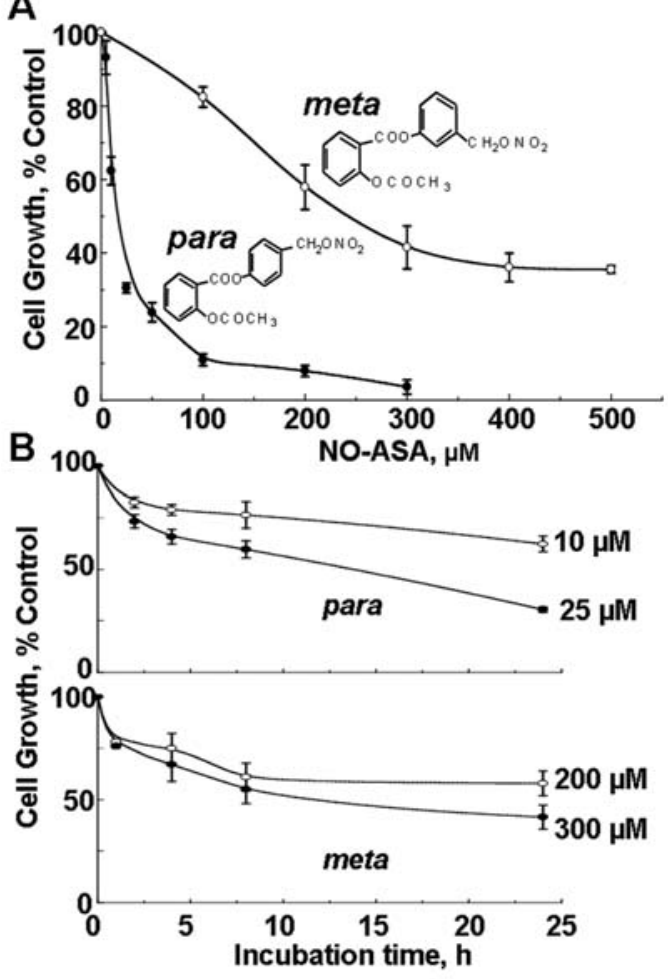

Figure 1. Effects of para and meta isomers of NO-ASA on cell growth. (A) HT-29 colon cancer cells were incubated for $24 \mathrm{~h}$ with various concentrations of either the para (black dots) or the meta (white dots) isomer of NO-ASA. Cell growth was determined by the MTT cell proliferation assay following the manufacturer's protocol. The structures of the positional isomers are shown next to the isomer's name. (B) Cells incubated for 1, 4, 8 and $24 \mathrm{~h}$ with concentrations below and above the $\mathrm{IC}_{50}$ for each isomer of NO-ASA. Data are expressed as the percent of untreated control cultures and standard error bar represent the standard error of the mean (SEM) for at least three separate experiments.

for up to $24 \mathrm{~h}$. Shown in Fig. 1A, are the dose response curves for growth inhibition by these isomers of NO-ASA. The $\mathrm{IC}_{50} \mathrm{~S}$ for the para and meta isomers differ by about 20 -fold, being $12 \pm 5$ and $230 \pm 15 \mu \mathrm{M}$, respectively. As shown in Fig. 1B, the time-response curves of both isomers follow a similar course: after an initial strong decline during the first $4 \mathrm{~h}$, there is a shallow decrease in the number of viable cells. Although the reason for this biphasic response is unclear, it is conceivable that it reflects the rate of metabolic transformation of NO-ASA (22).

The effect of $m$ - and p-NO-ASA isomers on MAPK activation. Treatment of colon cancer cells with $m$ - or $p$-NO-ASA stimulates MAPK phosphorylation over the $24 \mathrm{~h}$ incubation period. The effects we obtained, depicted in Fig. 2, can be summarized as follows.

At $1 \mathrm{~h}, p$-NO-ASA increased the phosphorylation of ERK1/2 MAPK significantly only at concentrations above its $\mathrm{IC}_{50}$ for cell growth, while at the concentrations used the meta isomer only demonstrated increased phosphorylation of ERK1/2 after $24 \mathrm{~h}$ treatment. Akt was marginally, if at all, affected by $p$-NO-ASA. At $\mathrm{IC}_{50}$ concentrations only the meta isomer significantly affected the phosphorylation of Akt, increasing it at 4 and $8 \mathrm{~h}$. The level of phosphorylated Akt returned to basal levels by the end of the $24 \mathrm{~h}$ incubation.
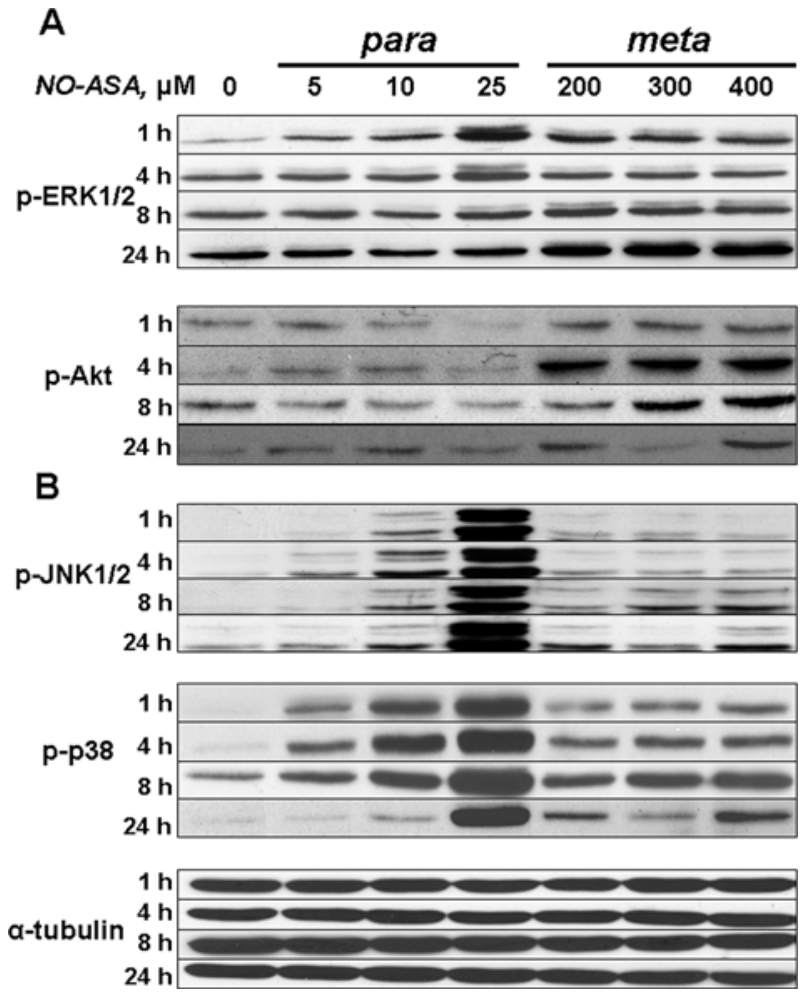

Figure 2. Effects of para and meta isomers of NO-ASA on MAPKs phosphorylation. HT-29 cells were treated with the indicated concentrations of each NO-ASA isomer for 1-24 h. Western blots of the phosphorylated forms of (A) ERK1/2 and Akt and (B) JNK and p38. $\alpha$-tubulin is shown as a loading control. For each time point comparisons should be made against the control (0) which was electrophoreticaly separated on the same polyacrylamide gel and transferred to the same membrane as the treated lysates for the same incubation period. Representative Western blots of more than five experiments are shown.

Both isomers of NO-ASA significantly increased the phosphorylation of the two stress-MAPKs, JNK and p38. Increased phosphorylation of JNK $1 / 2$ was observed within $1 \mathrm{~h}$ after treatment with $p$-NO-ASA and peaked at $4 \mathrm{~h}$, being approximately 10-fold higher than the basal level. Between 8 and $24 \mathrm{~h}$, the para isomer sustained an increased level of phosphorylated JNK of 2-3-fold over the basal level. At a concentration of $25 \mu \mathrm{M}$ the para isomer stimulated JNK phosphorylation to levels that were more than 20 -fold over basal phosphorylation. $m$-NO-ASA increased JNK phosphorylation, which peaked $8 \mathrm{~h}$ after treatment was initiated and was 3 -fold higher than the basal level. This phosphorylation was sustained for the entire period of observation (24 h).

Both isomers of NO-ASA stimulated large increases in the level of phosphorylated p38, starting as early as within $1 \mathrm{~h}$ of treatment. Similar to the stimulated phosphorylation of JNK, the para isomer at $25 \mu \mathrm{M}$ enhanced p38 phosphorylation by more than 20 -fold, maintaining that high level from 1-24 h. Compared to control baseline, $p$-NO-ASA $10 \mu \mathrm{M}$ (approximately at its $\mathrm{IC}_{50}$ ) stimulated p38 phosphorylation 8 -fold, an effect that peaked at $4 \mathrm{~h}$, slowed to 3 -fold over basal at 8 and at $24 \mathrm{~h}$ the level of phosphorylated p38 returned to near basal. Compared to control, the meta isomer at its $\mathrm{IC}_{50}$ increased p38 phosphorylation about 3-fold, peaking at $8 \mathrm{~h}$. As was observed with the para isomer, $m$-NO-ASA at 

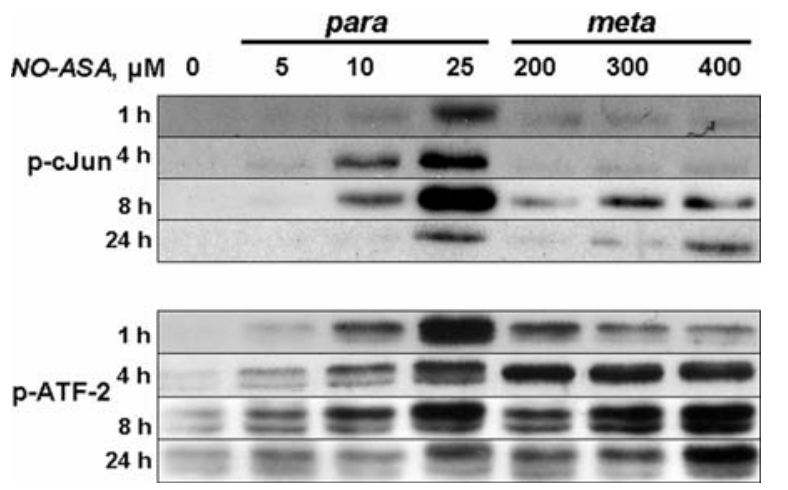

Figure 3. Effects of para and meta isomers of NO-ASA on the phosphorylation of MAPK transcription factors. HT-29 cells were treated with the indicated concentrations of each NO-ASA isomer for 1-24 h. Western blots of the phosphorylated forms of cJun and ATF-2. For each time point, comparisons should be made against the control (0) which was electrophoreticaly separated on the same polyacrylamide gel and transferred to the same membrane as the treated lysates for the same incubation period.

approximately twice its $\mathrm{IC}_{50}$ stimulated the prolonged phosphorylation of p38 that remained 5 to 8 -fold higher than its basal levels.

Given that the activation of JNK and p38 MAPKs leads to the activation of the downstream transcription factors cJun and ATF-2, we next evaluated if the isomers of NO-ASA activated these transcription factors. At $1 \mathrm{~h}, p$-NO-ASA $25 \mu \mathrm{M}$ activated cJun. This increased at later time points, becoming evident even at lower concentrations and peaking at $8 \mathrm{~h}$. At its highest ( $8 \mathrm{~h}, 25 \mu \mathrm{M} p$-NO-ASA), phosphorylated cJun was 10-12-fold higher than its basal level (Fig. 3). In contrast, the meta isomer at about its $\mathrm{IC}_{50}$ increased the phosphorylation of cJun much less and later than the para isomer (3-fold increase over baseline at its peak, which occurred at $8 \mathrm{~h}$ ). Both the para and meta isomers of NO-ASA at concentrations above their respective $\mathrm{IC}_{50} \mathrm{~s}$ maintained some residual cJun phosphorylation at $24 \mathrm{~h}$.

Both NO-ASA isomers phosphorylated ATF-2 early (within $1 \mathrm{~h}$ ) and fairly strong. As shown in Fig. 3 and 9, at concentrations approximately their $\mathrm{IC}_{50}$ values, the activation of ATF-2 in HT-29 cells was similar for both isomers, whereas at much higher concentrations, the para isomer $(25 \mu \mathrm{M})$ caused a strong and early activation of ATF-2 with meta $(400 \mu \mathrm{M})$ showing a later response, which appeared quantitatively similar to that of para.

In $\mathrm{BxPC} 3$ cells (which, in contrast to HT-29, could be transfected with siRNA), the strong activation of ATF-2 in response to $m$-NO-ASA appears to be partially regulated by p38 and JNK; silencing p38 or JNK or both reduces by about half the induction of ATF-2 by $m$-NO-ASA (Fig. 4A). Interestingly, $p$-NO-ASA $10 \mu \mathrm{M}$ does not induce ATF-2 in BxPC3 cells. Both $m$ - and $p$-NO-ASA induced the expression of c-Jun. Silencing JNK or JNK in combination with p38 virtually eliminated the induction of c-Jun by both NO-ASA isomers (Fig. 4B).

The effect of NO-ASA positional isomers on AP-1-DNA binding. We investigated if the differences in cJun and ATF-2 activation by the isomers of NO-ASA result in differences in the binding of these transcription factors to the AP- 1 consensus
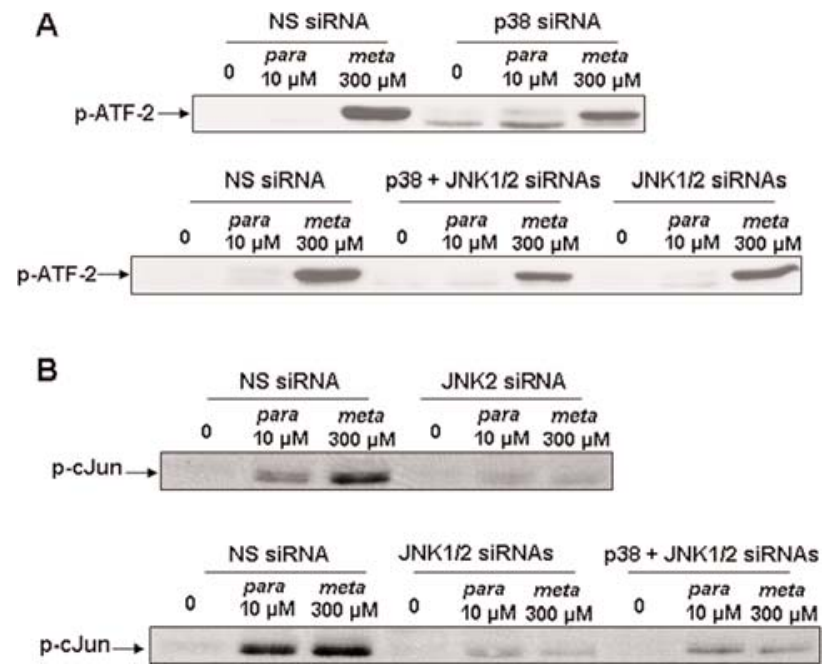

Figure 4. siRNA directed against JNK and p38 affects the activation of ATF and cJun by either isomer of NO-ASA. BxPC-3 cells were transfected with siRNA directed against p38 and/or JNK1/2 mRNA as described under Materials and methods before Western blotting. siRNA-treated and control cells were treated with para NO-ASA or meta NO-ASA (10 or $300 \mu \mathrm{M}$, respectively). Western blots for p-ATF-2 (A) and p-cJun (B) are shown. Representative Western blots of at least three independent experiments are shown.

sequence using EMSA. Supershift assays demonstrated that in these cells the AP-1 complex consisted of cJun and ATF-2 (data not shown). Both NO-ASA isomers increased the AP-1DNA binding. In keeping with the trend we have observed, the maximum AP-1-DNA binding effect for $p$-NO-ASA and $m$-NO-ASA was observed at 4 and 8 h, respectively (Fig. 5B). The EMSA blots were compared to Western blots of whole cell lysates from parallel cultures probed for the transcription factors. Interestingly, the binding of transcription factors in nuclear extracts to the AP-1 probe correlated with the phosphorylation level of cJun and ATF-2 (Fig. 5B).

The effect of NO-ASA positional isomers on p21 expression and cell cycle progression. A significant downstream effector of MAPKs is p21, a CDK inhibitor that limits proliferation by inhibiting cyclin-CDK complexes (25). We examined the effect of both isomers of NO-ASA on p21 levels in HT-29 cells. Both compounds induced the expression of p21 in a time- and concentration-dependent fashion (Fig. 6). In the case of $p$-NO-ASA, a strong and significant induction of $\mathrm{p} 21$ was noted at $4 \mathrm{~h}$, which dissipated by the end of the $24 \mathrm{~h}$ incubation. On the other hand, the meta isomer stimulated a much weaker increase in the levels of p21 that became apparent at $8 \mathrm{~h}$ and persisted at similar levels up to $24 \mathrm{~h}$ (Fig. 6).

We next analyzed the effect of each NO-ASA isomer on the cell cycle distribution of HT-29 cells. At an early time point $(4 \mathrm{~h})$, and consistent with the observed increase in p21 levels, only $p$-NO-ASA affected the cell cycle, blocking the S-to- $\mathrm{G}_{2} / \mathrm{M}$ transition (Table I). At $24 \mathrm{~h}$, when the effect of $p$ NO-ASA on p21 had essentially disappeared only a slight perturbation in the cell cycle was detected. The meta isomer, which showed no effect on p21 or the cell cycle during the first $4 \mathrm{~h}$, blocked the $\mathrm{G}_{2} / \mathrm{M}$-to- $\mathrm{G}_{1}$ transition at $24 \mathrm{~h}$ (Table I), following the induction of p21 that started at $8 \mathrm{~h}$ (Fig. 6). 

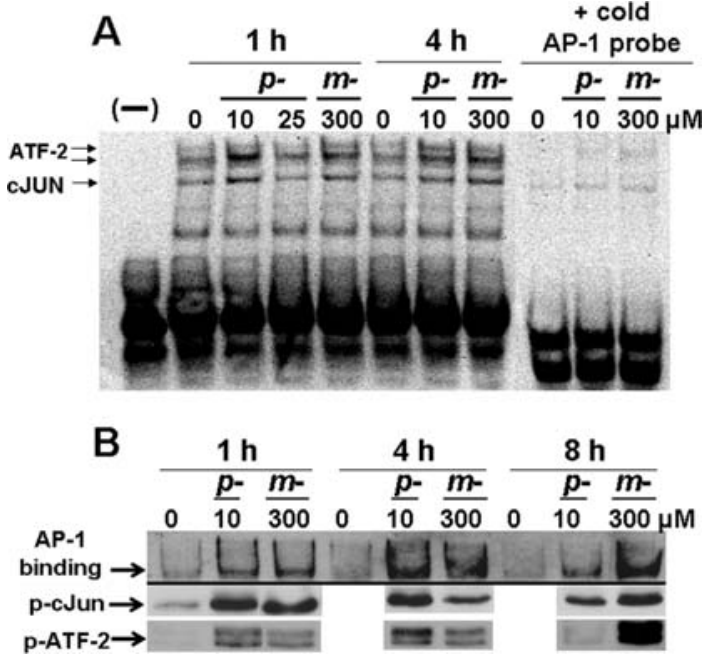

Figure 5. Effects of NO-ASA of transcription factor binding to the AP-1 complex. HT-29 cells were treated with the indicated concentrations of each NO-ASA isomer for 1-8 h. (A) EMSA for AP-1 in nuclear extracts isolated from control or treated HT-29 cells. (B) Non-denaturing gels were probed for cJun binding to the AP-1 probe and these were compared to Western blots of phosphorylated cJun and phosphorylated ATF-2 expression in whole cell lysates. Representative EMSA and Western blots of at least three independent experiments are shown.

To assess the relationship between MAPKs and p21, we investigated the effect of inhibiting p38 and JNK on p21. When p38 was inhibited by SB202190, the induction of p21 in response to either one of the NO-ASA isomers was reduced (Fig. 7A). In contrast, SP600125, the inhibitor of JNK, enhanced the induction of $\mathrm{p} 21$ by $p$-NO-ASA and to a much lesser degree by $m$-NO-ASA. These findings were assessed further by knocking down the expression of p38 and JNK using siRNAs. As shown in Fig. 7B, when the expression of p38 was knocked down in BxPC-3 cells, both $m$ - and $p$-NOASA failed to induce p21 (HT-29 cells are resistant to transfection with these siRNAs) Knocking-down the expression of JNK enhanced the expression of $\mathrm{p} 21$ in response to $p$-NOASA but the induction of $\mathrm{p} 21$ in response to $m$-NO-ASA was lost, indicating the opposite roles of JNK in signaling by these two isomers. Interestingly, the combined suppression of p38 and JNK by the respective siRNAs essentially eliminated the induction of p21 by either isomer (Fig. 7B).

Control experiments demonstrated that siRNA directed against p38 reduced p38 levels to $19 \%$ of those in response to non-specific siRNA. Also following the combined insertion of JNK1/2- and p38-directed siRNAs, the expression of total p38 was lower than $5 \%$ of the control. Similar results were obtained after transfection with siRNA directed against JNK $1 / 2$ or with both JNK1/2- and p38-directed siRNAs, where the expression of JNK $1 / 2$ was clearly reduced compared to controls, especially its lower band (data not shown).

Gene silencing of p21 or MAPKs blocks the suppression of cell growth by NO-ASA. BxPC-3 cells transfected with siRNA directed at $\mathrm{p} 38$ or JNK1/2 alone failed to alter the effect of $p$-NO-ASA on cancer cell growth (Fig. 8). However, silencing both $\mathrm{JNK} 1 / 2$ and $\mathrm{p} 38$, or $\mathrm{p} 21$, inhibited significantly the effect of $p$-NO-ASA on cell growth. In contrast, silencing of only $\mathrm{p} 38, \mathrm{JNK} 1 / 2$ or $\mathrm{p} 21$ reduced the effect of the meta isomer

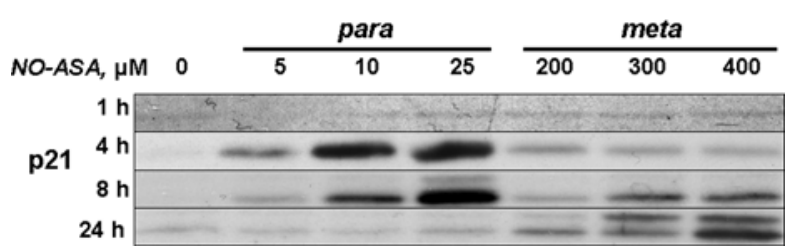

Figure 6. Effects of para and meta isomers of NO-ASA on p21 protein levels. HT-29 cells were treated with the indicated concentrations of each NO-ASA isomer for 1-24 h. Western blots for p21 are shown. For each time point, comparisons should be made against the control (0) which was electrophoreticaly separated on the same polyacrylamide gel and transferred to the same membrane as the treated lysates for the same incubation period. Representative Western blots of at least three independent experiments are shown.
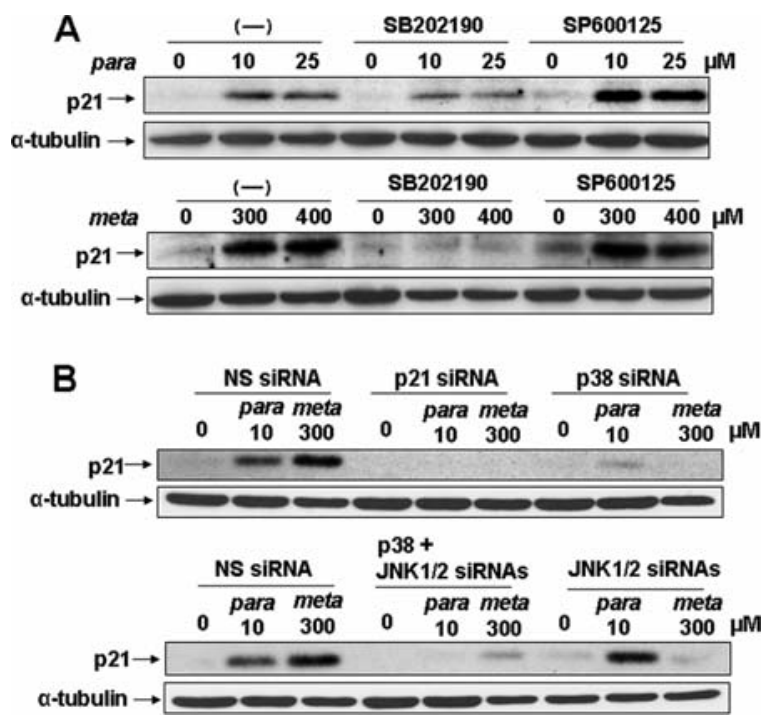

Figure 7. Effects of JNK and p38 inhibition on the induction of p21 expression. (A) HT-29 cells were treated with MAPKinhibitors SB202190 (p38) or SP600125 (JNK) both at $25 \mu \mathrm{M}$ for $30 \mathrm{~min}$ before further incubation with of either NO-ASA isomer for $8 \mathrm{~h}$. Western blots for p21 are shown. (B) BxPC-3 cells were transfected with siRNA directed against p21, p38 and/or JNK mRNA as described under Materials and methods before Western blotting. Western blots for $\mathrm{p} 21$ are shown. $\alpha$-tubulin is shown as a loading control. Representative Western blots of at least three independent experiments are shown.

on cell growth, as did the combined silencing of p38 and JNK1/2 (Fig. 8). These results indicate that $\mathrm{p} 21$ is a pivotal target for both isomers. However, p38 and JNK1/2, both upstream of $\mathrm{p} 21$, play different roles in the effect of the two NO-ASA isomers on cell growth. Whereas both p38 and $\mathrm{JNK} 1 / 2$ are required for the effect of $p$-NO-ASA on cell growth; silencing either p38 or JNK $1 / 2$ alone is enough to rescue the growth inhibitory effect of the meta isomer.

The differential signaling events of the two positional isomers of NO-ASA. To summarize our findings on NO-ASA signaling, we have divided the effects of the two isomers into early (1-4 h) and late (8-24 h) effects. In addition, to provide an overall semiquantitative assessment of the effect of each isomer during these two time periods, we assigned an overall value according to the intensity of the effect. Fig. 9, illustrating what was detailed above, highlights the principal differences 
Table I. Effects of para and meta isomers of NO-ASA on the cell cycle.

\begin{tabular}{|c|c|c|c|c|c|}
\hline & \multirow[b]{2}{*}{ Control } & \multicolumn{2}{|c|}{$p$-NO-ASA } & \multicolumn{2}{|c|}{$m$-NO-ASA } \\
\hline & & $0.5 \times \mathrm{IC}_{50}$ & $1 \times \mathrm{IC}_{50}$ & $0.5 \times \mathrm{IC}_{50}$ & $1 \times \mathrm{IC}_{50}$ \\
\hline \multicolumn{6}{|l|}{$4 \mathrm{~h}$} \\
\hline $\mathrm{G}_{0} / \mathrm{G}_{1}$ & 40.0 & 42.9 & 43.1 & 41.6 & 39.4 \\
\hline S & 32.2 & 32.4 & 35.3 & 31.5 & 33.2 \\
\hline $\mathrm{G}_{2} / \mathrm{M}$ & 27.8 & 24.6 & 21.7 & 26.7 & 27.5 \\
\hline \multicolumn{6}{|l|}{$24 \mathrm{~h}$} \\
\hline $\mathrm{G}_{0} / \mathrm{G}_{1}$ & 57.2 & 55.2 & 53.4 & 51.6 & 48.1 \\
\hline $\mathrm{S}$ & 20.0 & 21.9 & 22.4 & 25.0 & 18.3 \\
\hline $\mathrm{G}_{2} / \mathrm{M}$ & 21.9 & 22.1 & 23.3 & 22.0 & 31.4 \\
\hline
\end{tabular}

HT-29 cells were incubated for 4 or $24 \mathrm{~h}$ with $0.5 \mathrm{x}$ or $1 \times \mathrm{IC}_{50}$ of either the para or the meta isomer of NO-ASA. DNA content was determined from PI fluorescence as in Materials and methods. Percentages of the distribution of cells in $\mathrm{G}_{0} / \mathrm{G}_{1}, \mathrm{~S}$ and $\mathrm{G}_{2} / \mathrm{M}$ phases are shown. Values are representative of three independent experiments; results were within $10 \%$.

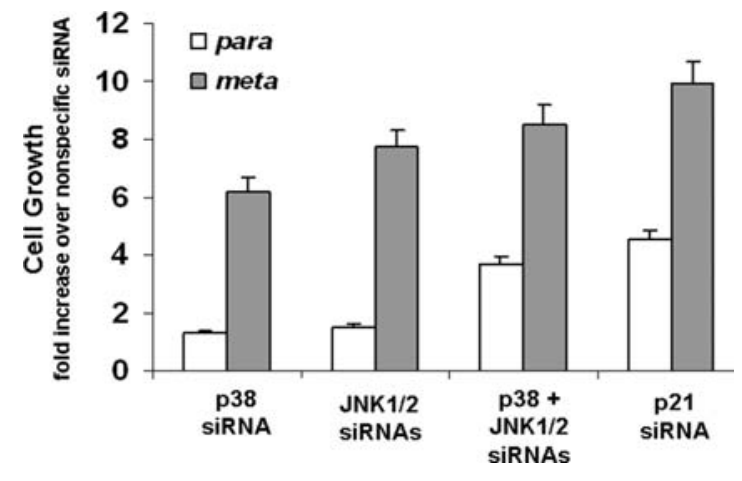

Figure 8. Effect of silencing JNK1/2, p38 or p21 on the growth inhibitory effect of NO-ASA. BxPC-3 pancreatic cancer cells were transfected with siRNA directed against p21, JNK1/2 and/or p38 mRNA. Forty-eight hours after transfection, cells were replated on 96-well plates and treated with either isomer of NO-ASA for $24 \mathrm{~h}$ before MTT assay was preformed. Data are expressed as the fold increase over the cell growth of non-specific siRNA control for each treatment.

between the two isomers: a) $p$-NO-ASA exerts its effect earlier than $m$-NO-ASA; b) the predominant effect of $m$-NO-ASA is on ERK $1 / 2$ and Akt; whereas that of $p$-NO-ASA is on JNK1/2, while both activate $\mathrm{p} 38$, with $p$-NO-ASA showing a stronger and earlier effect; c) ATF-2 is more responsive to $m$-NO-ASA and c-Jun to $p$-NO-ASA; d) both isomers seem to have similar effects on AP-1 binding, the main difference between them being the timing of the effect; $p$-NO-ASA's effect is early and $m$-NO-ASA's is late; e) $p$-NO-ASA has an earlier and stronger effect on p21, while $m$-NO-ASA's effect occurs later and is weaker and f) cell cycle changes follow the effect on p21 expression.

\section{Discussion}

Isomerism can influence drug activity, at times profoundly (13-15). Our study, focused on the $m$ - and $p$-positional isomers of NO-ASA, establishes a striking difference in biological activity between them. Their most important difference

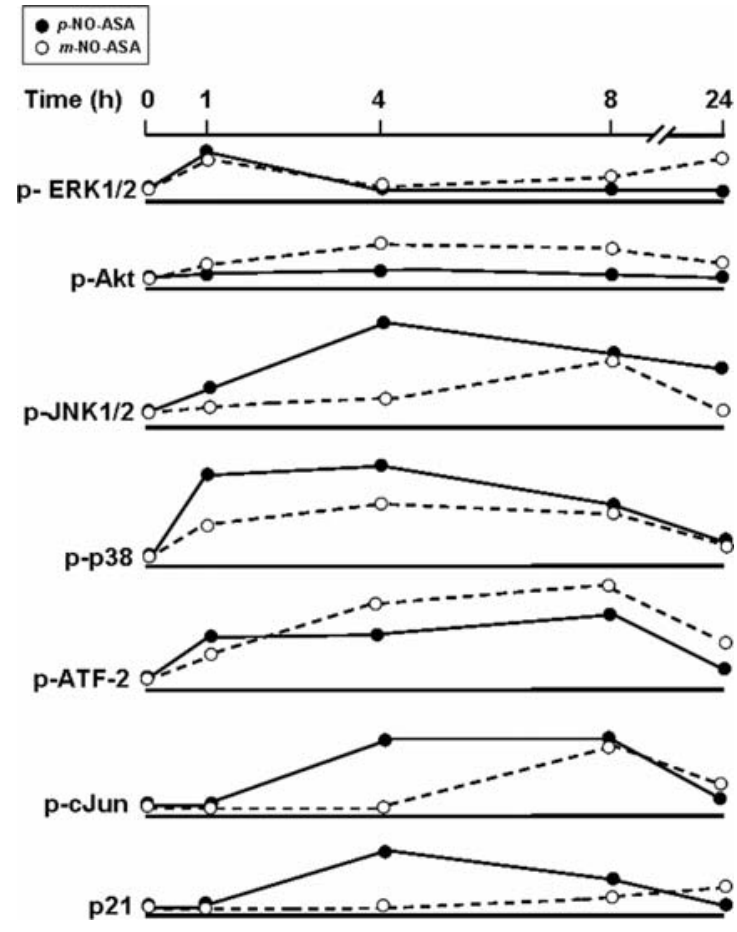

Figure 9. Scheme showing the differences in the effects on MAPK signaling triggered by either isomer of NO-ASA. The time response during which each change occurred and a rough representation of their relative intensity based on the quantification of the shown data. To note, the difference in the intensity and the time response for each particular protein activation or level measured is shown. The para NO-ASA $(10 \mu \mathrm{M})$ time responses for each parameter measured are shown in full lines with full dots, while the time responses of meta NO-ASA $(300 \mu \mathrm{M})$ are shown in broken lines with empty dots.

concerns their ability to inhibit colon cancer cell growth, with their $\mathrm{IC}_{50}$ values differing by about 20 -fold $(m-\mathrm{NO}-\mathrm{ASA}=$ $230 \pm 15 \mu \mathrm{M} ; p$-NO-ASA $=12 \pm 5 \mu \mathrm{M})$. The signaling studies that we performed provide at least a partial explanation of this dramatic difference in potency between the two positional isomers. 
Both NO-ASA isomers differentially affected the three individual pathways of the MAPK signaling cascade and the Akt pathway. The Akt kinase participates in the inhibition of the intrinsic apoptotic pathway, by phosphorylating Bad, which prevents its translocation to the mitochondria. $p$-NOASA failed to activate Akt, as evidenced by the absence of change of the levels of its phosphorylated forms, while $m$ NO-ASA activated Akt, its effect peaking at $4 \mathrm{~h}$. Although, the summation of the signaling effects of the meta isomer results in cell growth inhibition, the increased activation of Akt suggests an early anti-apoptotic response. However, this anti-apoptotic effect is overwhelmed by the later growth inhibitory events induced by $m$-NO-ASA.

NO-ASA isomers had little effect on ERK1/2 activation. The para isomer at its highest concentration activated ERK1/2 rapidly, while the meta isomer activated ERK1/2 modestly at $24 \mathrm{~h}$. It is unlikely, however, that the ERK1/2 pathway mediates the growth inhibitory effect of NO-ASA, especially for $p$-NO-ASA, since we have previously shown that inhibition of ERK1/2 activation by the ERK1/2 inhibitor PD98059 had no effect on the ability of $p$-NO-ASA to inhibit cell growth (19).

p-NO-ASA activated JNK and p38 along with their respective downstream transcription factors, cJun and ATF-2. The activation of these two MAPKs was quantitatively pronounced; the phosphorylation of p38 increased within the first hour of treatment and continued to rise at least for $8 \mathrm{~h}$ at all concentrations (and for $24 \mathrm{~h}$ at its highest concentration). These changes were accompanied by changes in the levels of phosphorylated ATF-2, which also increased rapidly after $p$-NO-ASA treatment. On the other hand, $m$-NO-ASA increased p38 phosphorylation more gradually than the para isomer, peaking at $8 \mathrm{~h}$. Moreover, the response to the meta isomer was weaker than to the para, leading to a later and modest increase in ATF-2 phosphorylation.

The phosphorylation of both isoforms of JNK, JNK1 and $\mathrm{JNK} 2$, increased rapidly in response to $p$-NO-ASA, reaching essentially maximal levels by $1 \mathrm{~h}$. Of note, treatment with the meta isomer, led to a delayed and weaker induction of phosphorylation of the JNK isoforms, peaking after $8 \mathrm{~h}$ of treatment. Activation of phosphorylated cJun by the activated JNK was evident after $8 \mathrm{~h}$ for both NO-ASA isomers. Whereas $p$-NO-ASA increased cJun phosphorylation strongly and progressively during the first $8 \mathrm{~h}$, the analogous effect of the meta isomer was only modest in intensity. This finding is in agreement with the degree of JNK1/2 phosphorylation that each isomer induced.

p21, a key signaling downstream molecule, was activated in response to MAPK activation, ultimately leading to cell cycle deceleration and cell growth inhibition. The differences in the time course for JNK and p38 MAPK activation by either NO-ASA isomer, were reflected in the induction of p21 protein expression. The expression of the cyclin-dependent kinase inhibitor $\mathrm{p} 21$, which blocks cell cycle progression by inhibiting the activity of cyclin/Cdk2 complexes (26-28), was differentially upregulated by the NO-ASA isomers. Whereas $p$-NO-ASA induced the rapid and strong expression of p21 in a dose- and time-dependent manner, the $m$-NO-ASA gradually induced a weaker p21 expression, peaking at $24 \mathrm{~h}$-treatment. This differential effect was also evident in the way these isomers arrested the cell cycle. While the para isomer blocked the cell cycle as early as $4 \mathrm{~h}$, the meta isomer arrested the cell cycle later (detected at $24 \mathrm{~h}$ ). The cell cycle effect by the NOASA isomers was clearly correlated with p21 levels.

The specific inhibition of JNK using a pharmacological inhibitor failed to block the effect of both NO-ASA isomers on p21 induction, but the inhibition of p38 did block the effect of either $p$ - or $m$-NO-ASA on p21 induction. This finding suggested that the $\mathrm{p} 38$ signaling cascade mediates the effect of both NO-ASA isomers on p21 induction. This conclusion was, however, tentative because it was based on the use of pharmacological inhibitors, which always have the potential of effects beyond their target proteins. The studies using either siRNA for gene silencing were, therefore, needed for a thorough assessment of the contribution of p38 and JNK. Whereas transfections with siRNA directed at JNK1/2 alone failed to alter the effect of either para or $m$-NO-ASA on p21 induction, the silencing of p38 alone or the combined silencing of JNK $1 / 2$ and p38 inhibited significantly the effect of both NO-ASA isomers on p21 induction. This finding confirms that p38 MAPK mediates p21 induction by NO-ASA.

When we analyzed the effect of JNK and p38 pathway on cell growth, we observed that transfections with siRNA directed at p38 or JNK1/2 alone failed to alter the effect of $p$-NO-ASA on cancer cell growth. In contrast, the combined silencing of JNK1/2 and p38 inhibited significantly the effect of $p$-NO-ASA on cell growth. The silencing of p21 also blocked the cell growth inhibitory effect of both isomers. These findings, being in excellent agreement with the results obtained using pharmacological inhibitors, lead to the conclusion that $p$-NO-ASA needs to stimulate the p38 and JNK pathways to inhibit cell growth. On the contrary, $m$-NOASA needs to modulate either p38 or JNK to inhibit cell growth. p21 is a pivotal growth inhibitory target for both isomers.

The present structure-activity study underscores the complexity of the effects of NO-ASA isomers on cancer cell growth. We have previously shown that there is an excellent correlation between the initial rate of NO release by the different NO-ASA molecules (meta and para) and their cell inhibitory potencies (16). This finding emphasizes the contribution of NO to the biological effect of NO-ASA. However, regardless of these findings, the $-\mathrm{ONO}_{2}$ group may not be the sole determinant of the biological activity of NOASA. Interestingly, the spacer is also important for NOASA's effect $(29,30)$. It is conceivable that the two isomers generate different intermediates (quinone methide vs. a carbocation) leading to different biological responses (29). Furthermore, there are differences in the metabolism between the two isomers (22). These differences could contribute to the differential response of the cells to the two NO-ASA isomers. For example, both isomers of NO-ASA start with a common deacetylation step and end up with similar end products. Following cleavage of the ester bond between the two benzene rings, the para isomer release $\mathrm{NO}_{3}{ }^{-}$and form end products rapidly via a putative short-lived intermediate, whereas the meta isomer forms the stable (nitroxymethoxy) phenol, which releases $\mathrm{NO}_{3}{ }^{-}$and forms end products at a much slower rate (22). This may in part explain the different 
time-profiles of the MAPK activation and p21 induction observed between the two isomers.

In conclusion, our study provides clear evidence that positional isomerism influences in an important way the NOASA molecule with regard to the inhibition of colon cancer cell growth. Our data also demonstrate that both NO-ASA isomers activate MAPKs, which regulate the expression of p21 leading to the growth inhibitory effect of NO-ASA. However, there are differences between the two isomers in the timing and intensity of these 'downstream' effects and they correlate with the growth inhibitory effect of NO-ASA. Our findings have potentially important implications for understanding the mechanism of action of these and related chemoprevention agents (31) as well as for the design of chemoprevention approaches.

\section{Acknowledgements}

Grant support: NIH R01 CA10101902.

\section{References}

1. Jemal A, Siegel R, Ward E, Murray T, Xu J and Thun MJ: Cancer statistics, 2007. CA Cancer J Clin 57: 43-66, 2007.

2. Baron JA: What now for aspirin and cancer prevention? J Natl Cancer Inst 96: 4-5, 2004.

3. Farmer KC, Goulston K and Macrae F: Aspirin and nonsteroidal anti-inflammatory drugs in the chemoprevention of colorectal cancer. Med J Australia 159: 649-650, 1993.

4. Gupta RA and DuBois RN: Aspirin, NSAIDS, and colon cancer prevention: mechanisms? Gastroenterology 114: 1095-1098, 1998.

5. Laine L: The gastrointestinal effects of nonselective NSAIDs and COX-2-selective inhibitors. Semin Arthritis Rheum 32: 25-32, 2002.

6. Laine L: Gastrointestinal effects of NSAIDs and coxibs. J Pain Symptom Manage 25: S32-S40, 2003.

7. Fiorucci S, Santucci L, Gresele P, Faccino RM, Del Soldato P and Morelli A: Gastrointestinal safety of NO-aspirin (NCX-4016) in healthy human volunteers: a proof of concept endoscopic study. Gastroenterology 124: 600-607, 2003.

8. Williams JL, Borgo S, Hasan I, Castillo E, Traganos F and Rigas B: Nitric oxide-releasing nonsteroidal anti-inflammatory drugs (NSAIDs) alter the kinetics of human colon cancer cell lines more effectively than traditional NSAIDs: implications for colon cancer chemoprevention. Cancer Res 61: 3285-3289, 2001.

9. Williams JL, Kashfi K, Ouyang N, del Soldato P, Kopelovich L and Rigas B: NO-donating aspirin inhibits intestinal carcinogenesis in Min (APC(Min/+)) mice. Biochem Biophys Res Commun 313: 784-788, 2004.

10. Williams JL, Nath N, Chen J, et al: Growth inhibition of human colon cancer cells by nitric oxide (NO)-donating aspirin is associated with cyclooxygenase- 2 induction and beta-catenin/Tcell factor signaling, nuclear factor-kappaB, and NO synthase 2 inhibition: implications for chemoprevention. Cancer Res 63: 7613-7618, 2003.

11. Zhou H, Huang L, Sun Y and Rigas B: Nitric oxide-donating aspirin inhibits the growth of pancreatic cancer cells through redox-dependent signaling. Cancer Lett 273: 292-299, 2009.

12. Ouyang N, Williams JL, Tsioulias GJ, et al: Nitric oxide-donating aspirin prevents pancreatic cancer in a hamster tumor model. Cancer Res 66: 4503-4511, 2006.
13. Ali I, Hussain I, Saleem K, Aboul-Enein HY and Bazylak G: Supramolecular chiro-biomedical assays and enantioselective HPLC analyses for evaluation of profens as non-steroidal antiinflammatory drugs, potential anticancer agents and common xenobiotics. Curr Drug Discov Technol 5: 105-120, 2008.

14. Constantinou C, Papas A and Constantinou AI: Vitamin E and cancer: An insight into the anticancer activities of vitamin $\mathrm{E}$ isomers and analogs. Int J Cancer 123: 739-752, 2008.

15. Raz A: Is inhibition of cyclooxygenase required for the antitumorigenic effects of nonsteroidal, anti-inflammatory drugs (NSAIDs)? In vitro versus in vivo results and the relevance for the prevention and treatment of cancer. Biochem Pharmacol 63: 343-347, 2002.

16. Kashfi K, Borgo S, Williams JL, et al: Positional isomerism markedly affects the growth inhibition of colon cancer cells by nitric oxide-donating aspirin in vitro and in vivo. J Pharmacol Exp Ther 312: 978-988, 2005.

17. Rigas B: The use of nitric oxide-donating nonsteroidal antiinflammatory drugs in the chemoprevention of colorectal neoplasia. Curr Opin Gastroenterol 23: 55-59, 2007.

18. Gao J, Liu X and Rigas B: Nitric oxide-donating aspirin induces apoptosis in human colon cancer cells through induction of oxidative stress. Proc Natl Acad Sci USA 102: 17207-17212, 2005.

19. Hundley TR and Rigas B: Nitric oxide-donating aspirin inhibits colon cancer cell growth via mitogen-activated protein kinase activation. J Pharmacol Exp Ther 316: 25-34, 2006.

20. Nath N, Kashfi K, Chen J and Rigas B: Nitric oxide-donating aspirin inhibits beta-catenin/T cell factor (TCF) signaling in SW480 colon cancer cells by disrupting the nuclear beta-cateninTCF association. Proc Natl Acad Sci USA 100: 12584-12589, 2003.

21. Williams JL, Ji P, Ouyang N, Liu X and Rigas B: NO-donating aspirin inhibits the activation of NF-kappaB in human cancer cell lines and Min mice. Carcinogenesis 29: 390-397, 2008.

22. Gao J, Kashfi K and Rigas B: In vitro metabolism of nitric oxide-donating aspirin: the effect of positional isomerism. J Pharmacol Exp Ther 312: 989-997, 2005.

23. Hundley TR, Gilfillan AM, Tkaczyk C, Andrade MV, Metcalfe DD and Beaven MA: Kit and FcepsilonRI mediate unique and convergent signals for release of inflammatory mediators from human mast cells. Blood 104: 2410-2417, 2004.

24. Hurst HC, Masson N, Jones NC and Lee KA: The cellular transcription factor CREB corresponds to activating transcription factor 47 (ATF-47) and forms complexes with a group of polypeptides related to ATF-43. Mol Cell Biol 10: 6192-6203, 1990.

25. Junttila MR, Li SP and Westermarck J: Phosphatase-mediated crosstalk between MAPK signaling pathways in the regulation of cell survival. FASEB J 22: 954-965, 2008.

26. Pei XH and Xiong Y: Biochemical and cellular mechanisms of mammalian CDK inhibitors: a few unresolved issues. Oncogene 24: 2787-2795, 2005.

27. Santamaria D and Ortega S: Cyclins and CDKS in development and cancer: lessons from genetically modified mice. Front Biosci 11: 1164-1188, 2006.

28. Weiss RH: p21Waf1/Cip1 as a therapeutic target in breast and other cancers. Cancer Cell 4: 425-429, 2003.

29. Hulsman N, Medema JP, Bos C, et al: Chemical insights in the concept of hybrid drugs: the antitumor effect of nitric oxidedonating aspirin involves a quinone methide but not nitric oxide nor aspirin. J Med Chem 50: 2424-2431, 2007.

30. Kashfi K and Rigas B: The mechanism of action of nitric oxidedonating aspirin. Biochem Biophys Res Commun 358: 1096-1101, 2007.

31. Zhao W, Mackenzie GG, Murray OT, Zhang Z and Rigas B: Phosphoaspirin (MDC-43), a novel benzyl ester of aspirin, inhibits the growth of human cancer cell lines more potently than aspirin: a redox-dependent effect. Carcinogenesis 30: 512-519, 2009. 